\title{
A Molecular Model for Singlet/Singlet Energy Transfer of Monovalent Ligand/Receptor Interactions
}

\author{
David L. Meadows* and Jerome S. Schultz ${ }^{\dagger}$ \\ Department of Chemical Engineering, The University of Michigan, \\ Ann Arbor, Michigan 48109-2136
}

Received April 26, 1990/Accepted September 11, 1990

A stochastic model is described that predicts the degree of singlet/singlet energy transfer in complexes formed between monovalent ligands and monovalent receptors. The modeling approach is intended to serve as an analytical tool for approximating the level of fluorescence quenching that can be expected to occur in fluorescently labeled monovalent ligands and receptors that are bound together in cornplexes. This approach has utility in areas such as modeling protein/protein interactions and designing fluorescence energy transfer assays.

Using the crystallographic data for papain (monovalent ligand) and concanavalin $A$ (monovalent receptor) along with a molecular graphics computational package, the ligand and receptor were docked together to form a ligand/receptor complex. The intermolecular distances between the lysine residues of the ligand and receptor were then estimated, and the average energy transfer for the docked ligand/ receptor complex was calculated assuming a value for the characteristic length $R_{0}$ of the donor/acceptor pair. Results from the stochastic model were used to calculate the level of fluorescence quenching one would expect for a resonance energy transfer competition assay based on the monovalent ligand/receptor pair.

Three key assumptions were made during the model development. First, all lysine residues for the ligand and receptor were equally reactive with the dye molecules so the stoichiometry of the donor and acceptor chromophores was governed by a binomial distribution. Second, the dye molecules were located at the $\alpha$-carbon position for each reactive lysine residue. Finally, in the energy transfer competition assay, it was assumed that equilibrium existed between the ligand, receptor, and competing hapten at all times. Based on these assumptions, results are presented that indicate the maximum energy transfer for the monovalent papain/concanavalin A complex is strongly dependent on the number of acceptor chromophores and on the value of $R_{0}$. Results are also presented on the approximate level of fluorescence quenching that may occur in a competition assay based on the papain/pConA complex. Lastly, a strategy is discussed for maximizing the dynamic range and linearity of energy transfer assays by optimizing several key design variables.

Key words: Resonance energy transfer - papain - concanavalin A - molecular model - monovalent ligand and receptor

\footnotetext{
* Present address: Allergan Pharmaceutical, 2525 Dupont Drive, Irvine, CA 92715.

${ }^{\dagger}$ To whom all correspondence should be addressed. Present address: Center for Biotechnology and Bioengineering, University of Pittsburgh, 911 William Pitt Union, Pittsburgh, PA 15260.
}

\section{INTRODUCTION}

Fluorescence spectroscopic techniques continue to be used in a wide range of biological applications such as microscopy, cell sorting, immunological assay development, macromolecular structure studies, etc. Many fluorescence assays have sensitivities that often approach the sensitivities of isotopic assays but without the inherent safety concerns that radioactive materials present. Intrinsic fluorescence from aromatic amino acids and/ or extrinsic fluorescence from dye/protein conjugates have been used as "spectroscopic rulers" for determining distances and shapes within single proteins and protein complexes in their native configuration. ${ }^{6,21-23}$ This approach is based on the phenomenon of resonance energy transfer between two complementary chromophores (a donor and acceptor) that are bound to the protein or protein complex. The accepted mechanism for the transfer process over distances typically ranging up to $70 \AA$ is the direct coupling of the emission transition dipole of the donor to the absorbance transition dipole of the acceptor. ${ }^{7}$

Several mathematical models have been developed over the past 20 years for predicting the effects of multiple donor/acceptor interactions on a single protein and in protein complexes. Since very little is usually known about the structure of the protein or protein complex, several assumptions are usually required. The most common assumptions are similar to those first outlined by Gennis and Cantor. ${ }^{9}$ First, all fluorescent dyes can be placed anywhere at random on the protein surface (nonspecific labeling). In other words, the dye molecules do not have to react with a specific set of amino acid residues. Next the stoichiometry of labeling is governed by a Poisson distribution. Finally, for protein complexes, the donors can be exclusively localized on the surface of one protein and the acceptors on another protein.

From these assumptions, Gennis and Cantor predicted the "anhydrous radius" of proteins and the distances between pairs of proteins in complexes. They noted the severe simplifications the assumptions imposed on a system, but for some systems, such as trypsin and $\alpha$-chymotrypsin and their protein inhibitors, "the experimentally observed energy transfer was in excel- 
lent agreement with expected values calculated from known protein radii". ${ }^{10}$ The Gennis/Cantor assumptions were used to model an antigen/antibody system, but much poorer results were obtained. ${ }^{24}$ For the antigen/ antibody system, the model correlated well with the actual experimental results only when about a third of the quenchers were associated with the $F_{A B}$ fragment of the antibody and the remaining quenchers were ignored. The authors concluded that "the Gennis and Cantor model must be used with caution." One of the primary reasons for the discrepancies between the model and experiments in the Gennis and Cantor model was that the quenchers could not in actuality be evenly distributed over the surface of the antibody. Instead, the quenchers were bound to specific sites, usually at lysine residues, that were not evenly distributed over the $F_{A B}$ and $F_{C}$ units.

To allow for some of the limitations in the Gennis and Cantor model, we have utilized a stochastic approach to demonstrate how resonance energy transfer can be modeled in a system containing two species (a receptor and a ligand) of monovalent proteins. Calculations of the energy transfer between two fluorescently labeled monovalent proteins were made using the actual $\mathrm{x}$-ray crystallographic data for the proteins. The advantage of using crystallographic data is that the donor and acceptor molecules can be attached to specific locations on a protein so that spatial relationship between the donors and acceptors for different orientations of the receptor/ligand pair is known. By modeling receptor/ ligand interactions using resonance energy transfer, we have shown how important orientation and affinity are for assays that involve macromolecular interactions. These factors have important implications when developing immunological assays with optimum sensitivities over an analyte concentration range of interest.

\section{THEORY OF NONRADIATIVE ENERGY TRANSFER}

According to Förster's theory, the efficiency of transfer between a single donor and acceptor, $E$, is given by

$$
E=r^{-6} /\left(r^{-6}+R_{0}{ }^{-6}\right) \quad 0 \leq E \leq 1
$$

where $R_{0}$, the separation at which the transfer efficiency is $50 \%$, is

$$
R_{0}=9.7 \times 10^{3}\left(J K^{2} Q_{0} n^{-4}\right)^{1 / 6}
$$

The geometric variables in these expressions are $r$, the distance between the centers of the donor and acceptor chromophores, and $K^{2}$, the orientation factor for dipole/ dipole interaction $\left(0 \leq K^{2} \leq 4\right)$. The spectroscopic variables are $J$, the spectral overlap integral; $n$, the refractive index of the medium between the donor and acceptor; and $Q_{0}$, the quantum yield of the energy donor in the absence of the acceptor. The spectral overlap integral $J$ (in $\mathrm{cm}^{3} \mathrm{M}^{-1}$ ) is a measure of the spectral overlap of the donor emission spectrum and the accep- tor excitation spectrum defined by

$$
J=\frac{\int F(\lambda) \varepsilon_{A}(\lambda) \lambda^{4} d \lambda}{\int F(\lambda) d \lambda}
$$

where $F(\lambda)$ is the fluorescence intensity per unit wavelength interval (arbitrary units) of the energy donor at wavelength $\lambda$ (in centimeters) and $\varepsilon_{A}(\lambda)$ is the extinction coefficient (in $\mathrm{cm}^{-1} M^{-1}$ ) of the energy acceptor. For efficient transfer of energy from the donor to the acceptor, the value of $R_{0}$ must be of the same magnitude or larger than $r$, which implies that the spectral overlap integral must be relatively large. Also, given the sixthpower dependence between $E$ and $r, E$ is sensitive to changes in $r$ only over a range of $0.5 R_{0}<r<1.9 R_{0}$. The theory developed by Förster has been tested experimentally, ${ }^{22}$ and the exponent on the separation $r$ appearing in the transfer efficiency $E$ [Eq. (1)] was determined to be $-5.9 \pm 0.3$. This is in excellent agreement with the exponent predicted by Förster.

Several of the donor/acceptor pairs commonly used for energy transfer studies are given in Table I along with some important spectral properties. In the table $R_{0}\left(\frac{2}{3}\right)$ indicates that $K^{2}=\frac{2}{3}$ has been used for these calculations, which allows for random angular orientation between the dipoles of the donor and acceptors. ${ }^{23}$ As evidenced in Table I, it is possible to study interactions in a wide range of biological systems by carefully selecting an appropriate donor/acceptor pair. For example, proximity relationships have been made for three specific binding sites of bovine rhodopsin in digitonin solutions.$^{25}$ From these results, the authors were able to hypothesize that rhodopsin acts as a light-controlled gate. As another example among many, the distance between the hydrophobic binding site and the thiol group required for carbohydrate binding in lima bean lectin has also been measured. ${ }^{14}$

\section{MODEL DESCRIPTION}

Understanding the mechanisms of receptor/ligand binding is very important in areas such as immunology, but the molecular interactions are often too complicated to model in a mechanistic fashion. For this reason, we have chosen to model a simplified system based on a saccharide binding protein, concanavalin A (ConA), as the receptor and a functionalized papain derivative as the ligand. Energy transfer can be used to determine the ratio of bound vs. free ligand by attaching a donor chromophore to the ligand and an acceptor chromophore to the receptor. There are several reasons for choosing the ConA/papain system. First, the chemical and physical properties of ConA and papain have been very thoroughly studied, and the $x$-ray crystallographic coordinates are resolved to at least $2 \AA$ for both proteins. Second, ConA is a very good protein for studying 
Table I. Resonance energy transfer properties for several common donor/acceptor pairs.

\begin{tabular}{llcccc}
\hline Donor & \multicolumn{1}{c}{ Acceptor } & $Q_{d}$ & $J, \times 10^{15}\left(\mathrm{M}^{-1} \mathrm{~cm}^{3}\right)$ & $R_{0}\left(\frac{2}{3}\right), \AA$ & References \\
\hline 1,5-DPE & EPE & 0.37 & 236 & 48.7 & 8 \\
2,5-DPE & EPE & 0.76 & 154 & 51.2 & 8 \\
2,6-DPE & EPE & 0.71 & 33.1 & 39.1 & 8 \\
2,6-DPE & 2,5-DPE & 0.71 & 1.3 & 22.8 & 8 \\
FAD & Eosin Maleimide & 0.2 & 24.4 & 44 & 20 \\
Dansyl & FITC & $0.1-0.2$ & $79-159$ & $33-41$ & 6 \\
Dansyl & RBITC & $0.1-0.2$ & 31 & $28-31$ & 6 \\
FITC & RBITC & 0.5 & 50 & 40 & 6 \\
FITC & FITC & 0.5 & 19 & $25-30$ & 13 \\
FITC & TRITC & 0.34 & 290 & 56 & 13 \\
FITC & TRITC & - & - & 50 & 24 \\
FCA & MCF & 0.5 & - & 57 & 15 \\
\hline
\end{tabular}

Abbreviations: DPE, dansylphosphatidylethanolamine; EPE, eosinphosphatidylethanolamine; FAD, flavin adenine dinucleotide; FCA, fluorescein-6-carboxylic acid; FITC, fluorescein isothiocyanate; RBITC, rhodamine B isothiocyanate; TRITC, tetramethylrhodamine isothiocyanate; MCF, 4',5'-dimethoxy-6-carboxyfluorescein.

the effects of polyvalence: its valence can be varied from 1 to 4 without allosteric interactions. Papain can be used as a monovalent fluorescent ligand for ConA by attaching a saccharide moiety to the cystine 25 active site and then attaching chromophores to the 10 lysine residues. With this system, the equilibrium binding constant between Con $A$ and papain can be varied by at least a factor of 17,000 using different saccharide moieties. $^{2}$ The model we have developed calculates the efficiency of energy transfer between a single protomer of ConA (pConA) labeled with the quencher and a papain molecule labeled with the donor and functionalized with a single saccharide moiety.

A molecular graphics package (MIDAS from the University of California at San Francisco) was used to dock a papain molecule to $\mathrm{pConA}$, and the papain was rotated $360^{\circ}$ in relation to the pConA about the bonding axis. The model is much like a dumbbell with one end free to rotate. The extent of acceptor labeling, the donor/acceptor pair, and the separation between pConA and papain were all varied to establish the range of energy transfer efficiencies expected for the entire ensemble of molecular interactions. No assumption was made in this approach about the general shape of the proteins, as was done in the Gennis and Cantor model, since the actual crystallographic coordinates were used.

\section{Concanavalin A Structure}

Unlike many lectins, ConA does not contain any prosthetic groups such as carbohydrates, lipids, or nucleic acids. Each intact protomer has 237 amino acid residues and is folded into a compact ellipsoidal dome, approximately $40 \times 39 \AA$ in cross section and $42 \AA$ high. Each protomer (MW 26,000) has one sugar binding site specific to glucose-, mannose-, or fructose-like saccharides and a metal binding site for $\mathrm{Mn}^{2+}$ and $\mathrm{Ca}^{2+}$, both of which are essential for sugar binding. At physiological $\mathrm{pH}, 7.4$, native Con $\mathrm{A}$ is composed of four identical subunits, and the sugar binding sites are noninteracting. Below pH 5.6, a pConA tetramer will separate into two identical dimers. The dimer/tetramer dynamic equilibrium is also affected by temperature. ${ }^{17}$

ConA was the first intact lectin for which the amino acid sequence was determined ${ }^{5}$ and the first lectin to have the three-dimensional structure resolved to $2.4 \AA$ using $x$-ray diffraction ${ }^{11,12}$ (Fig. 1). The ConA saccharide binding site is approximately $350-600 \mathrm{pm}$ wide, $750 \mathrm{pm}$ high, and $1.8 \mathrm{~nm}$ deep. By further refining the crystallographic data, ${ }^{1}$ the amino acid residues were located that define the saccharide binding site: $12-18$, 98-102, 205-208, and 226-229. Carver and co-workers were also able to identify the side-chain and backbone atoms responsible for favorable interactions with highaffinity ligands and those responsible for unfavorable interactions with low-affinity ligands.

The crystallographic model developed by Carver and co-workers helped to explain why and how the spatial orientation of simple sugars, oligosaccharides, and glycopeptides in the binding site is so important for ConA binding. Prior experimental work ${ }^{2,16}$ had shown that a

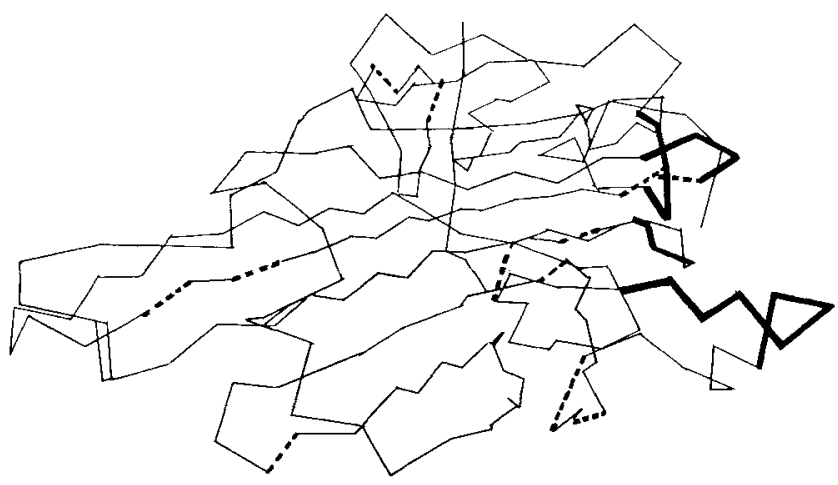

Figure 1. The x-ray crystallographic structure of the $\alpha$-carbons for Con A with the positions of the 12 lysine residues identified as dotted lines. The saccharide binding site is formed by amino acid segments highlighted with bold lines: $12-18,98-102,205-208$, and $226-229$. 
series of oligosaccharides and glycopeptides has very large differences in the inhibitory effects on ConA agglutination of red cells. Debray and co-workers demonstrated experimentally that by varying the residue sequence and the degree of branching, the inhibitory effects of polysaccharides could be more than 17,000 times greater than the inhibitory effects of glucose.

\section{Papain Structure}

Papain is a sulfhydryl protease that is able to hydrolyze peptide bonds behind 12 different amino acid residues. Papain can also function as an acyl-enzyme intermediate, a catalyst for hydrolysis of the $\mathrm{C}-\mathrm{X}$ bond, and a catalyst for a transacylation reaction. ${ }^{4}$ The single polypeptide chain of papain is 212 amino acid residues and is folded into two distinct parts which are divided by a cleft. Figure 2 gives the $x$-ray structure of papain with the dotted circle locating the sulfhydryl active site. ${ }^{3}$ Papain has three disulfide bonds and a sulfhydryl active site at cystine 25 . The slightly elongated shape is $50 \times 37 \times 37 \AA$ with an isoelectric point of $8.75 .^{18}$

\section{Assumptions}

The number of lysine residues was known for each protein, 12 for pConA and 10 for papain, and it was assumed that every lysine on a protein molecule had an equal probability of reacting with a dye. This assumption made it possible to use a binomial distribution for all statistical calculations. If $\mu$ is the average number of dyes per protein and $n$ is the total number of lysines, then the probability $P(x)$ of finding a protein with $x$ dyes (where $0 \leq x \leq n$ ) is

$$
P(x)=\frac{n !}{(n-x) ! x !} p^{x}(1-p)^{n-x}
$$

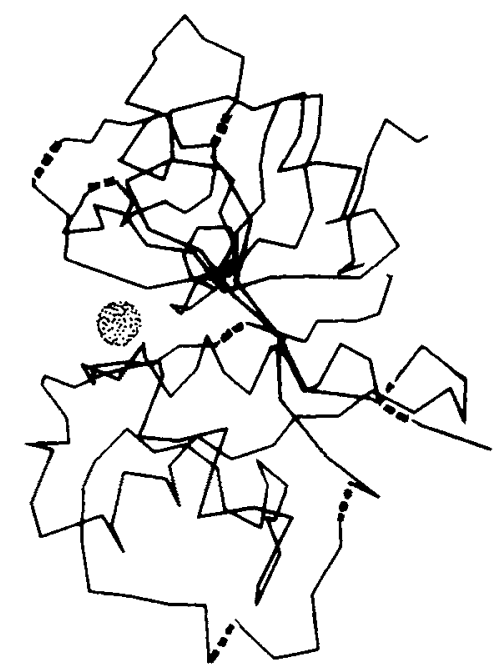

Figure 2. The x-ray crystallographic structure of the $\alpha$-carbons for papain with the positions of the 10 lysine residues identified as dotted lines. The position of the sulfhydryl binding site at cystine 25 is marked by the spotted circle. where $p$ is the probability of finding a protein with a dye, $p=\mu / n$. For low values of $\mu$, there will be a significant fraction of the protein molecules that are not labeled (e.g., 35\% for ConA when $\mu=1$ ). The impact of the large fractions of unlabeled protein on the level of energy transfer will be discussed later.

In all the calculations, dye molecules were assumed to be positioned at the $\alpha$-carbon of the lysine residue to which it is bound. This approximation was made because the exact location of each dye molecule was uncertain due to the freedom of movement each molecule could experience. The closest approach of pConA and papain was assumed to occur when the protein surfaces formed by the van der Waals forces came into contact (Fig. 3). Under these conditions, the minimum separation between the sulfhydryl site on papain and the center of the saccharide site on pConA was determined to be $18.4 \AA$ by connecting the spotted spheres in Figure 4 . In other words, a spacer arm connecting the papain sulfhydryl binding site with the saccharide moiety must be at least $18.4 \AA$ long for the pConA and papain to bind.

The average energy transfer between a papain molecule labeled with donors and a pConA molecule, $x$ lysines labeled with acceptors, was determined by allowing for relative rotation between the papain and pConA molecules. The axis of rotation was the line segment that joins the two binding sites shown in Figure 3. At each rotational increment $j$, through $360^{\circ}$, the efficiency for each donor in the pConA/papain complex was calculated as

$$
E_{k, j}=\frac{\sum_{i=1}^{x}\left(\frac{R_{0}}{r_{i, j}}\right)^{6}}{1+\sum_{i=1}^{x}\left(\frac{R_{0}}{r_{i, j}}\right)^{6}}
$$

for each possible arrangement $k$ of the $x$ acceptors among the 12 possible binding sites, where $r_{i, j}$ is the distance from the donor to the $i$ th acceptor and $R_{0}$ is assumed to be the same for all donor/acceptor pairs.' This is a good assumption for chromophores that are insensitive to changes in the microenvironment of the protein. Equation (5) assumes that energy transfer from a single donor to multiple acceptors occurs by parallel first-order kinetics. This also applies in the case where there is more than one donor on a papain molecule provided that the probability of two or more of the donors being excited simultaneously is negligible and there is no interdonor energy transfer. The average efficiency per donor is the arithmetic average of efficiencies from a single donor placed randomly at one of the 10 papain sites to $x$ acceptors placed randomly at the $12 \mathrm{ConA}$ sites. The average efficiencies are not dependent on the degree of donor labeling. After the efficiency was calculated for $x$ acceptors in all $k$ configurations for each rotation increment, the arithmetic average efficiency was calculated over all $360^{\circ}$ of rotation. This assumed that all rotational orientations were equally probable and 


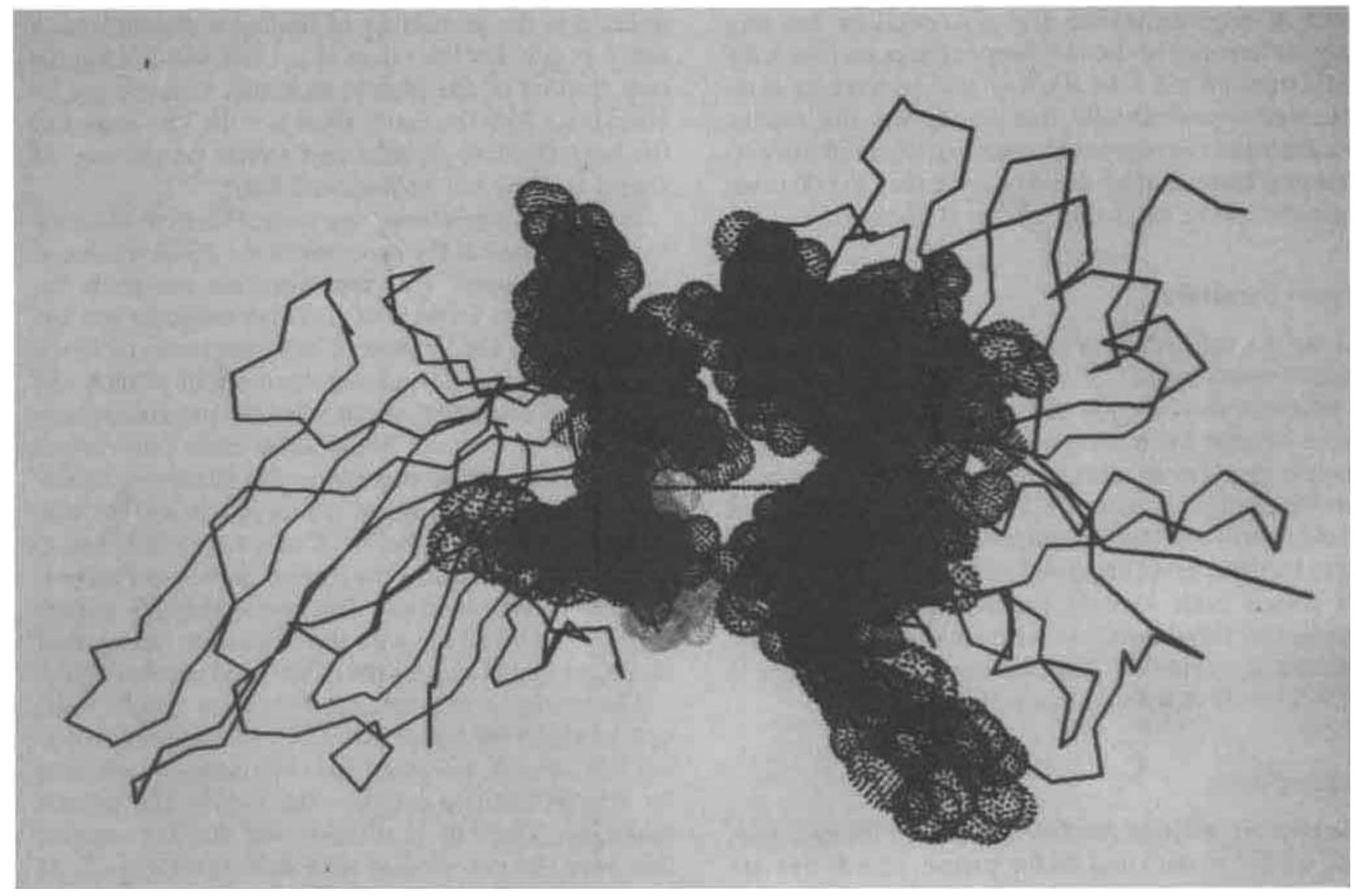

Figure 3. The closest approach of the ConA and papain is shown using the van der Waals volume (shown as spotted circles) of key regions of each protein. The spotted line segment joining the saccharide binding site of Con A with the sulf hydryl binding site for papain is $18.4 \AA$ long.

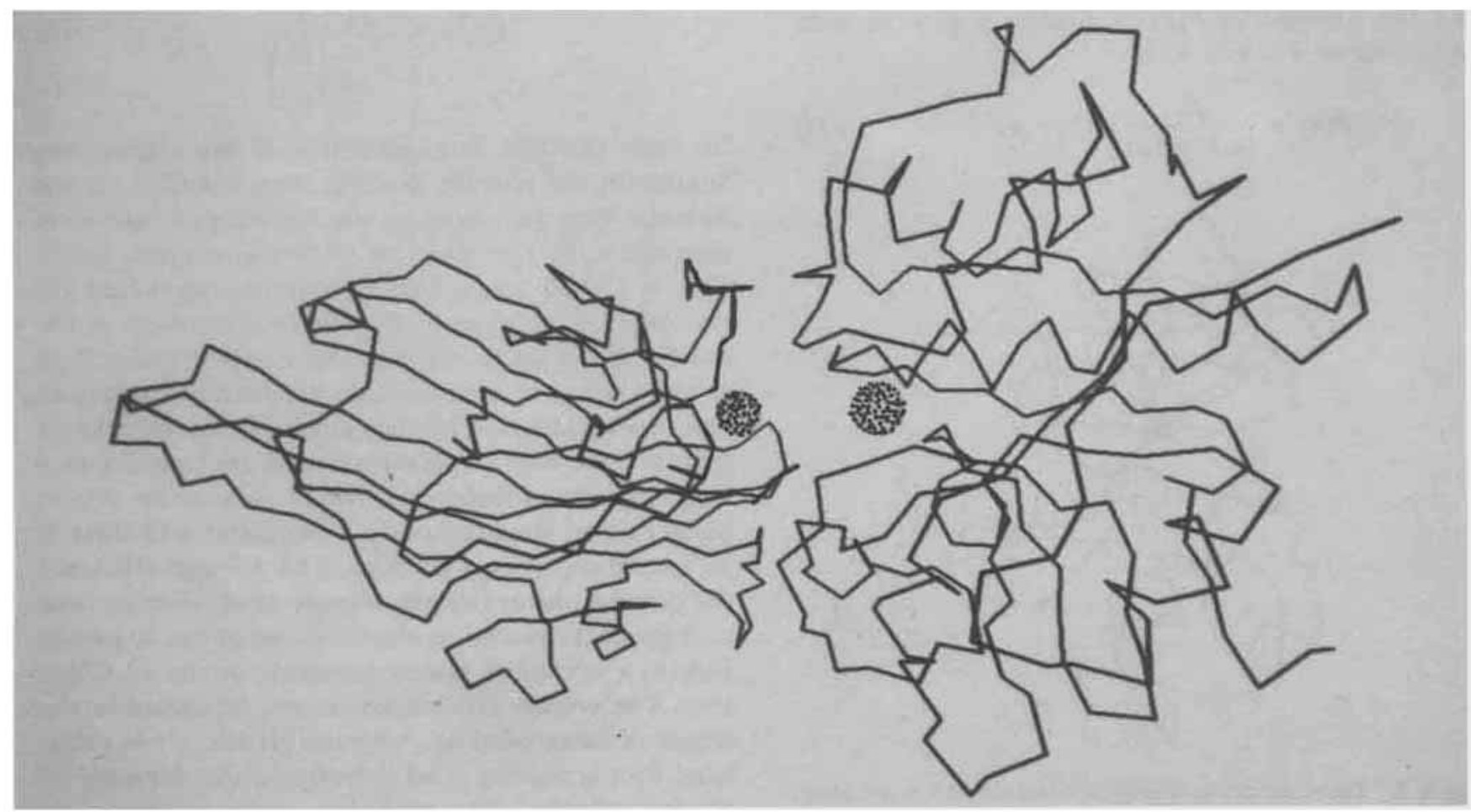

Figure 4. The orientation of ConA and papain at the closest approach without the van der Waals volumes being shown. The centers of the saccharide and sulfhydryl binding sites are highlighted as spotted circles. 
that, if they exchanged, the time scale of exchange was long compared with the transfer time. After completing these calculations over all $k$ for each $x(0 \leq x \leq 12)$ and over $360^{\circ}$ of rotation, the binomial distribution [Eq. (4)] was superimposed over the PConA population for given labeling ratios. The overall average energy transfer efficiencies were calculated for the whole ensemble. The stochastic calculations were continued for sufficient number of random $\mathrm{pConA} /$ papain labeling configurations for convergence. Normally, 1000 configurations were sufficient. Calculations were made following this procedure with different values of $R_{0}$ and with different distances separating the pConA and papain molecules.

Results from the stochastic model of energy transfer in a homogeneous solution containing papain-saccharide and $\mathrm{pConA}$ were then used in combination with a kinetic model for competitive binding developed by Schultz. ${ }^{19}$ In this model, a competing ligand (glucose) and a papain-saccharide molecule compete for the available pCon A binding sites. When the glucose concentration increases, a portion of the bound donorlabeled papain-saccharide molecules are displaced. This causes the energy transfer efficiency to decrease, resulting in an increase in donor fluorescence. The model assumes that only monovalent interactions occur between pConA and papain-saccharide and that equilibrium exists at all times. The reaction scheme can be written as

$$
\begin{array}{r}
\begin{array}{c}
\text { Glucose } \\
\text { (Analyte: A) }
\end{array} \\
\begin{array}{r}
\text { TRITC-pConA } \stackrel{K_{a}}{\longleftrightarrow} \\
\text { Glucose: TRITC-pConA } \\
\text { (Analyte: Receptor) }
\end{array} \\
\text { FITC-papain } \left.\quad+\text { TRITC-pConA } \mathrm{K}_{a}^{*}\right) \\
\text { (Competing analyte: } \left.\left.\mathrm{A}^{*}\right) \quad \text { (Receptor: } \mathrm{R}^{+}\right) \\
\text {FITC-Papain: TRITC-pConA } \\
\text { (Competing analyte: receptor) }
\end{array}
$$

where $K_{a}$ and $K_{a}^{*}$ are the equilibrium constants, $\mathrm{R}^{+}$represents acceptor-labeled pConA, and $\mathrm{A}^{*}$ represents donor-labeled papain that has been conjugated to a single saccharide haptenic moiety. Solving the reaction equations simultaneously, Schultz reduced the system down to a single dimensionless equation:

$$
\begin{aligned}
\left(\frac{\left[A^{*}\right]}{\left[A^{*}\right]_{t}}\right)^{2}+\left(\frac{\left[A^{*}\right]}{\left[A^{*}\right]_{t}}\right)\left[\frac{\left[R^{+}\right]_{t}}{\left[A^{*}\right]_{t}}-1+\frac{K_{a}[A]+1}{\left[A^{*}\right]_{t} K_{a}^{*}}\right] \\
-\frac{K_{a}[A]+1}{\left[A^{*}\right]_{t} K_{a}^{*}}=0
\end{aligned}
$$

where $\left[R^{+}\right]$and $\left[A^{*}\right]$ are the total concentrations of acceptor labeled pConA and donor-labeled papainsaccharide, respectively. Papain-saccharide and pConA molecules, which are unlabeled because of the stochastic nature of the labeling process, are also included in these concentrations. Using Equation (8) in combination with the energy transfer calculations for donor fluorescence quenching, it is possible to estimate the donor fluores- cence signal response for $\mathrm{pConA} /$ papain-saccharide interactions. This approach is sufficiently general to be applied to other reversible binding reactions where the $\mathrm{x}$-ray crystallographic coordinates are known for the receptor and ligand and may prove useful in designing assays based on resonance energy transfer.

\section{RESULTS}

A series of calculations have been performed that examine the effects of three variables on the efficiency of energy transfer in monovalent protein complexes: degree of acceptor labeling, characteristic length $R_{0}$, and separation between papain and pConA. All three of the variables can be adjusted experimentally to optimize the energy transfer efficiency for a particular system or application. The degree of labeling can be adjusted by the reaction conditions or the reactivity of the functional group. Length $R_{0}$ can be varied by changing the donor/ acceptor pair, and the separation between pConA and papain can be changed by varying the length of the tether connecting the saccharide moiety and the sulfhydryl active site on papain.

Results for the energy transfer efficiencies between one donor on papain and an average of $x$ acceptors on pConA are given for ensembles $A$ through $E$ in Figure 5. It is clear from these results that the number of acceptors strongly affects the efficiency of energy transfer for all cases. The actual energy transfer efficiencies used in computing the ensemble averages are shown in Figure 6. Comparison of Figures 5 and 6 shows that when the binomial population distribution was superimposed on the values in Figure 6, there was not a significant change in the shape of the curves for the ensemble population. Calculations for ensembles $A, D$, and $E$ used an $R_{0}$ value of $45 \AA$ for the donor/acceptor pair. The donor and acceptor that would have approximately this value of $R_{0}$ are fluorescein isothiocyanate and tetramethylrhodamine isothiocyanate (see Table I).

The maximum energy transfer efficiency for ensemble $A$ was approximately $93 \%$ when the average number of acceptors per pConA protomer was greater than 7. For low levels of acceptor labeling the efficiencies were much lower. A main contributing factor to the low efficiencies was the subpopulation of molecules in the binomial distribution that was not labeled with acceptors. When the $R_{0}$ value was decreased to $35 \AA$, the maximum efficiency was $77 \%$ for an average of 11 acceptors on the pConA receptor. Lowering $R_{0}$ still further to $25 \AA$ for ensemble $C$ gave a maximum efficiency of only $40 \%$. Ensemble $C$ demonstrates that when papain and pConA are bound together, over $60 \%$ of the donor fluorescence will not be quenched even when all of the lysines on pConA are occupied with an acceptor chromophore. The low quenching efficiency for ensemble $C$ will result in high background signals and low dynamic ranges for assays that use chromophores with 


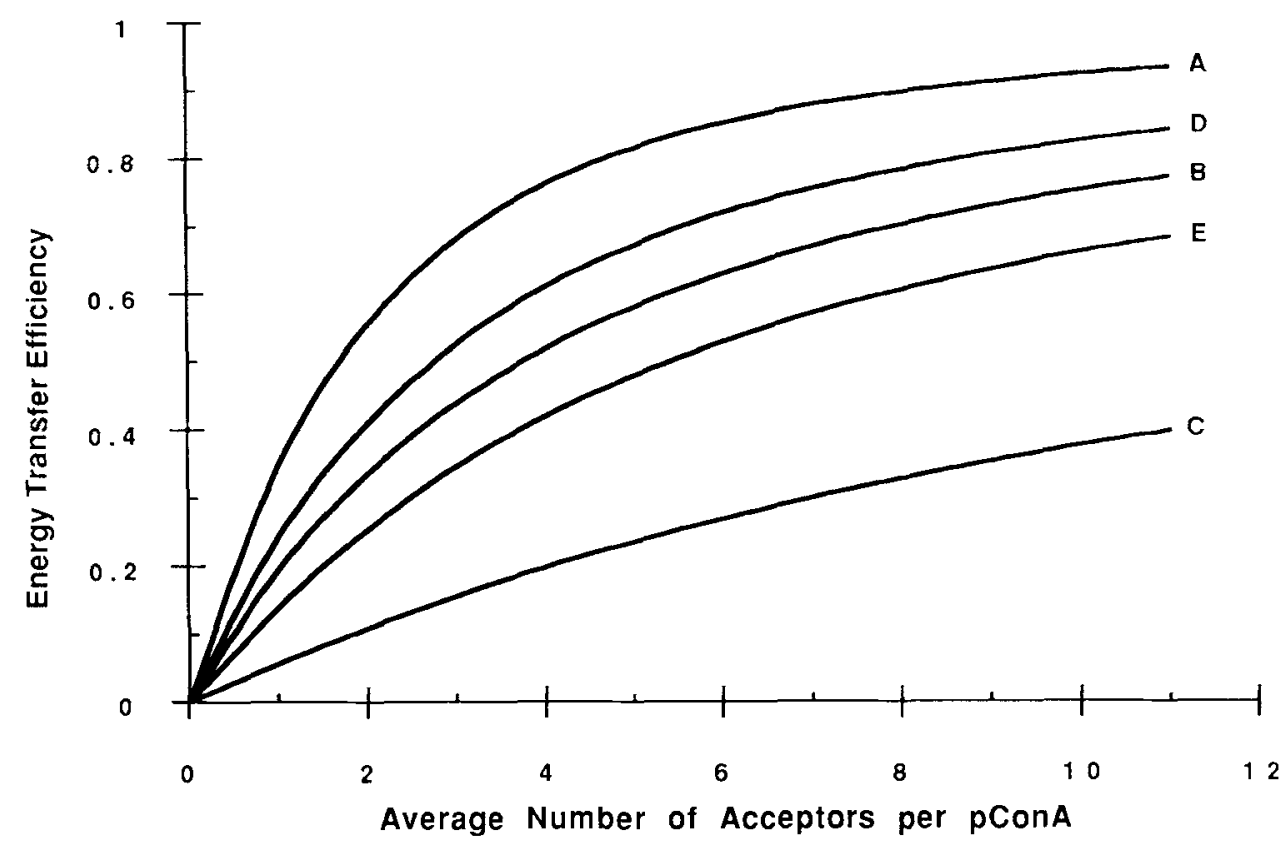

Figure 5. The average energy transfer efficiency for one donor as a function of the number of acceptors per ConA for ensembles $A-E$. Ensemble $A$ has $R_{0}=45 \AA$; ensemble $B$ has $R_{0}=35 \AA$; ensemble $C$ has $R_{0}=25 \AA$; ensemble $D$ has $R_{0}=45 \AA$ and a separation between molecules of $10 \AA$; ensemble $E$ has $R_{0}=45 \AA$ and a separation between molecules of $20 \AA$.

Transfer Efficiencies from One Donor in Papain/ConA Complex

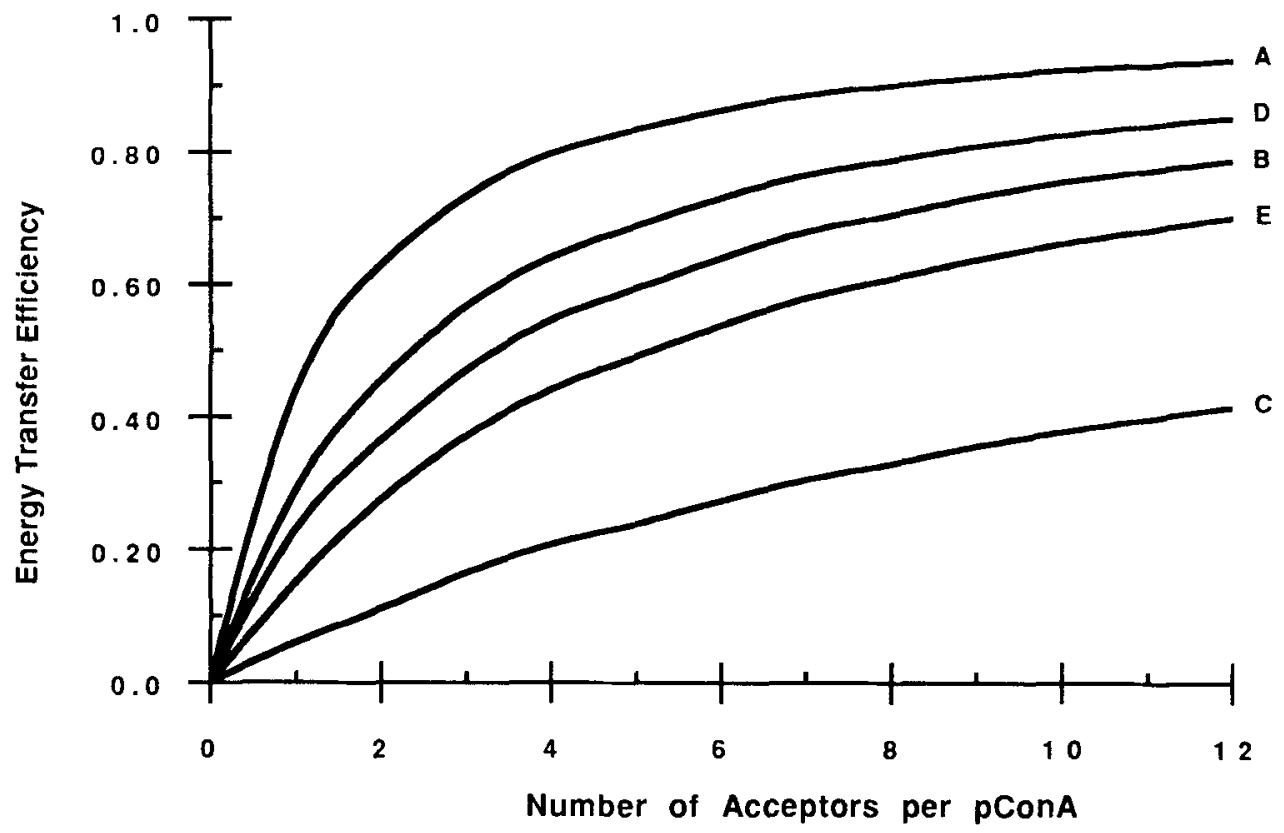

Figure 6. The average energy transfer efficiencies between a papain molecule with one donor and a ConA molecule with $x$ acceptors. The average efficiencies were calculated from all possible acceptor arrangements, $k$, and all rotational increments, $j$.

small $R_{0}$ values. This behavior has often been evidenced in experimental work with energy transfer assays. ${ }^{8}$

Another set of calculations was performed for the case when papain and PConA were separated by an additional $10 \AA$. These calculations were intended to esti- mate the effect of steric limitations between the ligand and receptor on the energy transfer efficiencies. As mentioned earlier, the closest approach of the papain and pCon $\mathrm{A}$ active sites was approximately $18.4 \AA$ when it was assumed that only van der Waals forces limited 
how closely the proteins could approach. The curve for ensemble $D$ in Figure 6 shows that increasing the separation to $28.4 \AA$ had very little effect on efficiency for an $R_{0}$ value of $45 \AA$. The maximum efficiency was still approximately $83 \%$. When the separation was increased to $38.4 \AA$, the maximum efficiency had decreased to $68 \%$ (ensemble $E$ ). Of course, for smaller $R_{0}$ values the efficiencies would show a much stronger dependence on the separation. The combined results for all five ensembles show that the energy transfer efficiency is more sensitive to the $R_{0}$ value of the donor/acceptor pair than it is to the separation between pConA and papain molecules.

The above results from the stochastic model were then combined with the kinetic model for unimolecular competing reactions described by Equations (6) and (7). Figure 7 is a plot of Equation (8) that shows the relation-

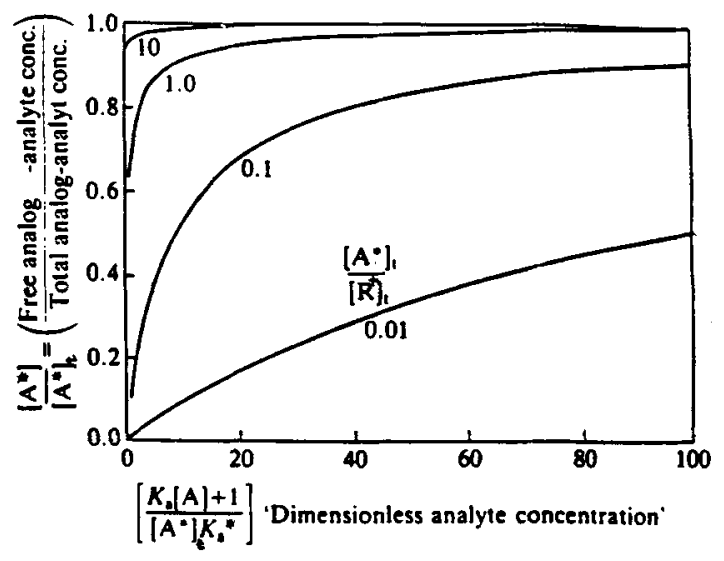

Figure 7. The change in the ratio of free to total analog analyte as a function of the dimensionless analyte concentration and the total analog analyte/total receptor ratio. ${ }^{19}$ ship between the dimensionless analyte concentration and the fraction of free analog/analyte. For the pConA/ papain system, Figure 7 shows how the ratio of free to total papain-saccharide is related to the dimensionless analyte (glucose) concentration for different ratios of total papain-saccharide to pConA. It is clearly seen in Figure 7 how important the proper kinetic parameters are for ensuring a large signal response over the analyte concentration range of interest. To illustrate the use of the figure, if the dimensionless analyte concentration range for a given application is between 0 and 40 , the $\left[A^{*}\right]_{t} /\left[R^{+}\right]_{t}$ ratio should be between 0.01 and 0.1 to achieve the largest signal response with the least nonlinear behavior. This is an important consideration when optimizing the design of assays based on resonance energy transfer (D. L. Meadows and J. S. Schultz, unpublished results). For example, when the $\left[A^{*}\right]_{t} /\left[R^{+}\right]_{t}$ ratio is 0.1 , the fraction of free papain-saccharide increases from 0.1 to 0.8 over a dimensionless concentration range of $1-40$. On the other hand, for an $\left[A^{*}\right] /\left[R^{+}\right]$ ratio of 10 , the fraction of free papain-saccharide goes from 0.95 to almost 1.0. These findings have a very strong impact on the dynamic range and the amount of donor quenching that is observed in a competitive binding assay based on resonance energy transfer.

Figures 8 and 9 present two examples of the combined results of the resonance energy transfer stochastic model and the competitive binding kinetic model. In Figure 8, an estimate is given of the donor fluorescence recovery over a dimensionless concentration range of 0-100 for energy transfer efficiencies of $0.2,0.4,0.6$, and 0.8 and an $\left[A^{*}\right] /\left[R^{+}\right]$ratio equal to 0.01 . Similar results are given in Figure 9 except that the $\left[A^{*}\right]_{t} /\left[R^{+}\right]_{t}$ ratio has been increased to 0.1 . The results in Figures 8

\section{Donor Fluorescence Recovery for a Ratio of 0.01}

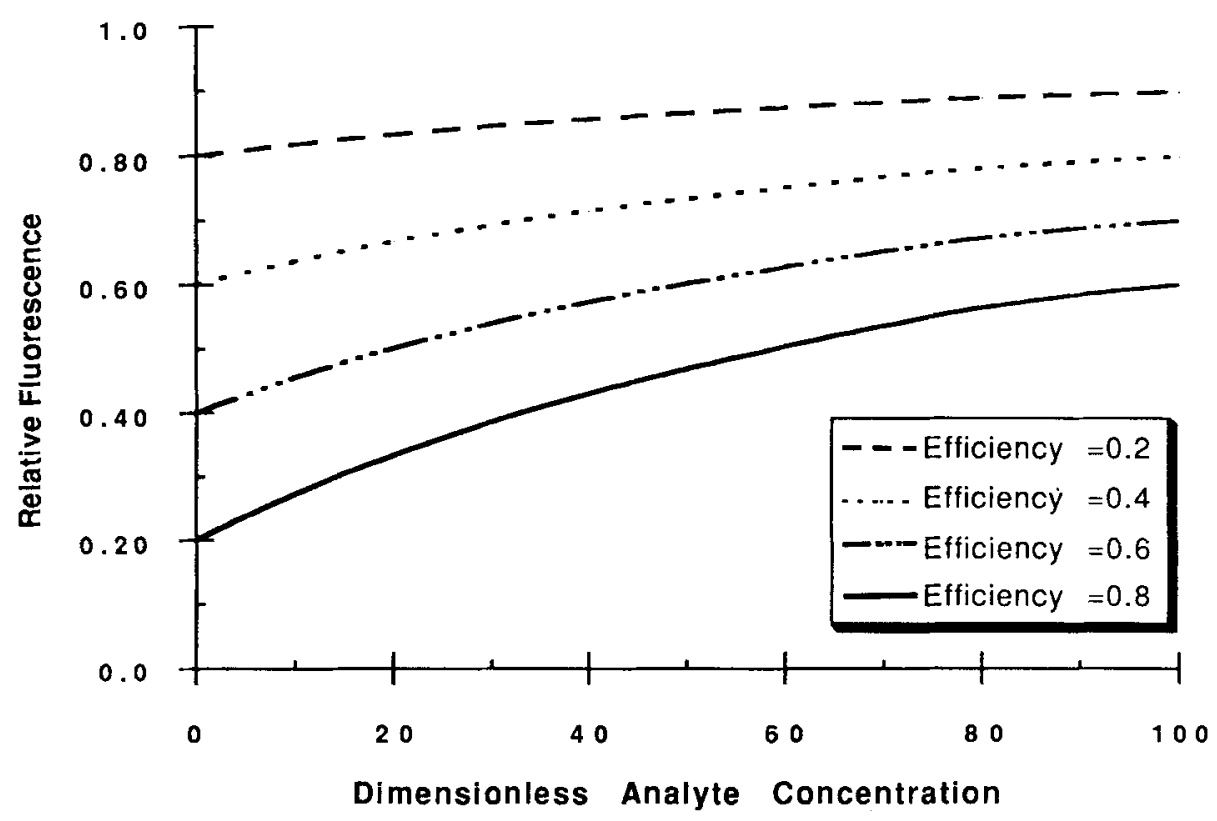

Figure 8. The effect of the dimensionless analyte concentration and energy transfer efficiency on the relative fluorescence when the total analog analyte/total receptor ratio is equal to 0.01 . 
Donor Fluorescence Recovery for a Ratio of 0.1

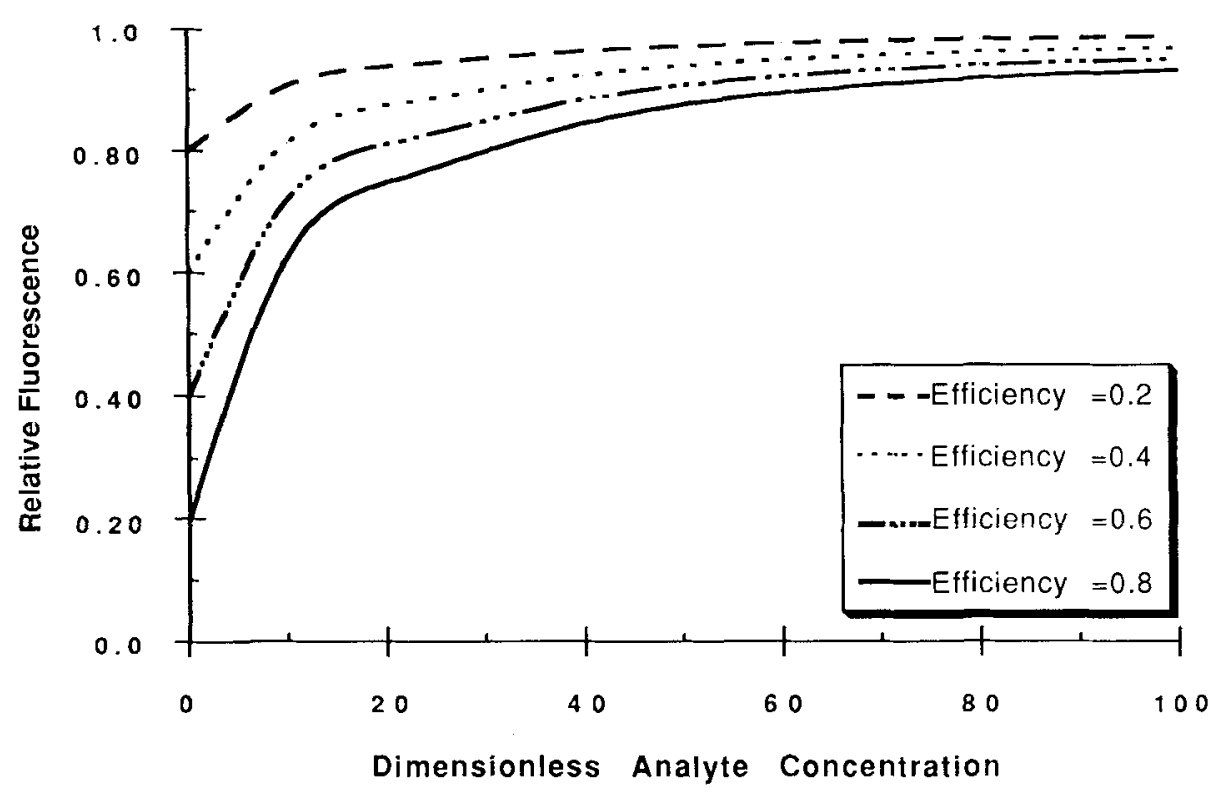

Figure 9. The effect of the dimensionless analyte concentration and energy transfer efficiency on the relative fluorescence when the total analog analyte/total receptor ratio is equal to 0.1 .

and 9 were obtained from Figure 7 as follows. The relative amount of fluorescence that is transferred from the donor to the quencher molecules in the bound complexes was calculated from the energy transfer efficiency and the bound fraction $\left[\left(1-\left[A^{*}\right] /\left[A^{*}\right]_{t}\right) \times\right.$ efficiency] which was then subtracted from 1.0 to give the final expression, $1-\left(1-\left[A^{*}\right] /\left[A^{*}\right]_{i}\right) \times$ efficiency.

Using this model can provide significant insights for the preliminary design of monovalent competitive binding systems. As an example, if the efficiency in Figure 9 were 0.4 , the relative fluorescence would increase from 0.6 to 0.9 over a dimensionless analyte concentration range of $0-40$. Assuming the ratio of $K_{a} / K_{a}^{*}$ was 1.0 and $\left[A^{*}\right]_{t}$ was $1 \mathrm{~m} M$, the analyte concentration range $[A]$ between 0 and $40 \mathrm{~m} M$ would correspond to a dimensionless concentration range between 0 and 40 . For a system composed of donor-labeled papain-saccharide and glucose that compete for binding sites on acceptor-labeled pConA, the relative fluorescence would increase from 0.6 to 0.9 over a glucose range of $0-40 \mu M$. This calculation assumes that the papain-saccharide ligand has a binding constant for pConA that is 1000 times stronger than the affinity of glucose for $\mathrm{pConA}\left(K_{a} / K_{a}^{*}=0.001\right)$. These examples briefly illustrate the utility of the mechanistic molecular model developed for the pConA/ papain-saccharide resonance energy transfer system.

\section{DISCUSSION}

The calculations reported here demonstrate that it is possible to estimate the level of fluorescence quenching that will occur in a homogeneous energy transfer assay system of a monovalent receptor and a monovalent macromolecular ligand. With the current state of the art in computer molecular modeling and the large crystallographic data base, it is possible to position the receptor and ligand molecules adjacent to one another and approximate the possible interaction configurations. Our calculations showed that energy transfer efficiencies approached $95 \%$ when the $R_{0}$ value was approximately equal to the dimensions of the proteins (approximately $45 \AA$ for the papain/pConA pair). The calculations also showed the importance of having a relatively large number of receptors if high efficiencies are to be obtained.

If donor/donor quenching were considered in the calculations, the efficiencies could be significantly higher for high donor loadings on the papain-saccharide ligand. The reason for this is that donor/donor interactions also follow parallel first-order kinetics which compete with the donor/acceptor interactions. However, when a photon is absorbed by a donor that is far away from an acceptor and yet is close to a donor, the photon can be shuttled to the second donor. If the second donor is closer to the acceptor, then the original photon can transfer to the acceptor through this shuttle mechanism. In Table $I$ the $R_{0}$ for FITC/FITC interactions is reported to be $25-30 \AA$ while the $R_{0}$ value for FITC/ TRITC interactions is approximately $50 \AA$. This means that if an FITC molecule were $20 \AA$ from another molecule of FITC, then the efficiency of transfer would be $87 \%$ while the efficiency of transfer between an FITC/TRITC pair would be $99 \%$. Further refinements can be made in the model if warranted by including the donor/donor interactions, but the complexity of the model would significantly increase if all of the secondary, tertiary, etc., interactions were included. 
If the donor/donor interactions were not desired, then interactions could be eliminated quite easily by using a donor with a larger Stokes shift than FITC. The larger Stokes shift would decrease the overlap of the absorbance and fluorescence spectra of the donor and lower the donor/donor transfer efficiency. An alternate way of significantly reducing the donor/donor interactions would be to replace papain with another proteolytic enzyme that has fewer lysine residues with which the donors can react. Also, the residues should be distributed throughout the protein so they are not located close together. A majority of the lysine residues must still be located close to the acceptors so efficient quenching by the acceptors can occur. Porcine elastase might be a good alternate because it has only three lysine residues. ${ }^{18} \mathrm{~A}$ final option for reducing the level of donor/donor interactions would be to substitute the lysine residues that are furthest away from the binding site, e.g., 10, 39, 100, 106, 190, and 211, with arginine residues by genetic engineering. As a result of these substitutions, only the four lysines nearest the binding site would be available for donor labeling.

This modeling approach is sufficiently general to be used for assay systems of other analytes, e.g., drugs, metabolites, and hormones. The ab initio requirement for the procedure is a bioreceptor that is specific to the analyte of interest and has a binding constant in the required range. For example, if a drug analog were covalently tethered to the sulfhydryl binding site on papain, then the energy transfer interactions could be modeled using existing $\mathrm{x}$-ray crystallography coordinates for papain and the coordinates for an antibody or an $F_{A B}$ fragment. More complex polyvalent receptor systems can also be modeled with this approach if the crystallography coordinates are available for the receptor.
We would like to thank Professor Martha Ludwig of the Biophysics Department at The University of Michigan for the generous use of her molecular graphics work station.

\section{References}

1. Carver, J. P., MacKenzie, A.E., Hardman, K.D. 1985. Biopolymers. 24: 49.

2. Debray, H. Decout, D. Strecker, G. 1981. Eur. J. Biochem. 117: 41.

3. Drenth, J. Jansonius, J. N., Koekoek, R. 1968. Nature. 218: 929.

4. Drenth, J., Jansonius, J. N., Koekoek, R. 1971. Adv. Protein Chem. 30: 79 .

5. Edelman, G. M., Cunningham, B.A., Reeke, G. N. 1972. Proc. Nat. Acad. Sci. 69: 2580.

6. Fairclough, R. H., Cantor, C. R. 1978. Meth. Enzymol. 48: 347.

7. Förster, T. 1960. Rad. Res. Supp. 2: 326.

8. Fung, B. K. K., Stryer, L. 1978. Biochem. 17: 5241.

9. Gennis, R. B., Cantor, C. R. 1972. Biochem. 11: 2509.

10. Gennis, L.S., Gennis, R. B., Cantor, C.R. 1972. Biochem. 11: 2517.

11. Hardman, K. D., Ainsworth, C. F. 1972. Biochem. 11: 4910.

12. Hardman, K. D., Ainsworth, C. F. 1976. Biochem. 15: 1120.

13. Johnson, D. A., Voet, J. G., Taylor, P. 1984. J. Biol. Chem. 259: 5717

14. Kella, N. K. D., Roberts, D. D., Shafer, J. A. 1984. J. Biol. Chem. 259: 4777.

15. Khanna, P. L., Ullman, E. F. 1980. Anal. Biochem. 108: 156.

16. Kornfeld, R., Ferris, C. 1975. J. Biol. Chem. 250: 2614.

17. McKenzie, G. H., Saw yer, W. H., Nichol, L.W. 1972. Biochim. Biophys. Acta. 263: 283.

18. Mihalyi, E. 1984. Application of proteolytic enzymes to protein structure studies, Vol. 1, CRC Press, Boca Raton, FL.

19. Schultz, J. S. 1987. Ann. NY Acad. Sci. 506: 406.

20. Scouten, W. H., Visser, A. J.W. G., Grande, H. J., 1980. Eur. J. Biochem. 112: 9.

21. Stryer, L. 1960. Rad. Res. Supp. 2: 432.

22. Stryer, L., Haugland, R. P. 1967. Proc. Nat. Acad. Sci. 58: 719.

23. Stryer, L. 1978. Ann. Rev. Biochem. 47: 819.

24. Ullman, E. F., Schwarzberg, M., Rubenstein, K. E. 1975. J. Biol. Chem. 251: 4172.

25. Wu, C.W., Stryer, L. 1972. Proc. Nat. Acad. Sci. 69: 1104. 